Revue d'histoire de l'enfance « irrégulière »

Le Temps de l'histoire

$14 \mid 2012$

Enfances déplacées. (I) en situation coloniale

\title{
Enfances colonisées
}

Une histoire postcoloniale des migrations juvéniles, XIXe-XXe siècles

David Niget

\section{(2) OpenEdition}

Journals

Édition électronique

URL : http://journals.openedition.org/rhei/3381

DOI : 10.4000/rhei.3381

ISSN : $1777-540 \mathrm{X}$

Éditeur

Presses universitaires de Rennes

Édition imprimée

Date de publication : 30 décembre 2012

Pagination : 35-44

ISBN : 978-2-7535-2194-0

ISSN : $1287-2431$

Référence électronique

David Niget, «Enfances colonisées », Revue d'histoire de l'enfance « irrégulière » [En ligne], 14 | 2012, mis en ligne le 30 décembre 2014, consulté le 02 mai 2019. URL : http://journals.openedition.org/ rhei/3381 ; DOI : 10.4000/rhei.3381

Ce document a été généré automatiquement le 2 mai 2019.

(c) PUR 


\section{Enfances colonisées}

Une histoire postcoloniale des migrations juvéniles, XIXe-XXe siècles

\section{David Niget}

\section{La colonisation comme utopie sociale}

1 Passées ses prémices guerrières, le projet colonial ne témoigne pas seulement d'une volonté de puissance, mais aussi de l'idée selon laquelle une nation peut engendrer, sur un territoire neuf, une société neuve, qui soit à la fois héritière de ses qualités, mais libre de ses déterminismes, de ses conflits de classe, des clivages politiques qui la travaillent et la figent. Aussi, l'enfance apparaitt-elle comme une page blanche sur laquelle les anciennes nations pourraient tracer cette utopie coloniale. La société coloniale serait une société de la seconde chance pour les fils et filles des "classes dangereuses" de la métropole, corrompues par l'industrialisation, une société qui permettrait aux enfants indigènes de s'extraire de leur "sauvagerie » pour connaître la " civilisation », mais ce, pour tous ces enfants, au prix du sacrifice de leurs racines. Car il s'agit bien alors de couper l'enfant de son environnement, de le transporter tantôt vers la colonie, tantôt vers la métropole pour en faire un homme neuf, un colon productif, un citoyen idéal.

2 Les parallèles entre l'enfant et le colon occupent d'emblée une grande place dans les discours philanthropiques sur la protection de l'enfance déshéritée. Le thème du « retour à la terre » est très présent dans la pensée philanthropique du XIX ${ }^{\mathrm{e}}$ siècle; de nombreuses utopies politiques, puissantes $\mathrm{du} \mathrm{XVII}^{\mathrm{e}}$ au XIX ${ }^{\mathrm{e}}$ siècle, accréditent l'idée de sociétés rurales préservées de la corruption morale. De la colonisation intérieure à la colonisation impériale, il n'y a qu'un pas. L'enfant pourra alors incarner l'archétype du colon, être vierge sur un territoire vierge, se fécondant mutuellement.

3 Avec le territoire, c'est l'enfance qu'il s'agit de coloniser au XIX ${ }^{\mathrm{e}}$ siècle, comme le rappelait Michelle Perrot dans un texte programmatique ${ }^{1}$. La protection de l'enfance s'est réalisée au prix de sa ségrégation, de son arrachement à un «milieu » jugé corrupteur, d'où la nécessité d'une migration forcée, d'un (dé)placement. Plus encore, cette protection s'est accomplie au prix de l'aliénation de l'enfant en tant que sujet. L'enfant, sous la plume des médecins, des pédagogues, des économistes du $\mathrm{XIX}^{\mathrm{e}}$ siècle, devient progressivement, un 
«autre ». Au fur et à mesure que l'on étudie l'enfant et que l'on marque sa spécificité physiologique, psychique, économique, on fabrique également sa différence. Et cette altérité qui le rend étranger au monde des adultes est décrite comme une sorte de sauvagerie, qui se cristallisera, au $\mathrm{xx}^{\mathrm{e}}$ siècle, dans la notion d'adolescence. Sous la plume du psychologue Granville Stanley-Hall, l'enfance récapitule l'évolution humaine, du primitif au civilisé. On perçoit bien ici à quel point les parallèles entre le sauvage et l'enfant sont prégnants, et comment le pouvoir colonial pourra alors s'appliquer à l'enfant en toute légitimité . Sauvage, l'enfant est un être à éduquer, par la force si nécessaire, tout comme l'indigène.

4 À cette conception de la malléabilité infantile mise au jour par les psychologues et les pédagogues répond la plasticité des sociétés coloniales, comme le montre Ellen Boucher dans son article. Dès lors, le projet colonial peut se donner pour mission de civiliser les enfants autochtones comme de dépayser les enfants de la métropole, selon les mêmes visées : engendrer un homme ou une femme nouveaux, produire une société neuve. Et selon le projet colonial, cette genèse se produit à la fois sous la contrainte, parfois arbitraire, d'un État qui s'exonère du droit lorsque nécessaire, mais aussi sous la bannière de la liberté, l'idéal colonial résidant dans la capacité des futurs colons à inventer leur avenir dans un espace libre, à constituer une société d'individus autonomes et productifs. C'est aussi en cela que la colonisation apparait comme une utopie pédagogique : diriger l'enfant au nom de sa protection, contraindre et émanciper à la fois, nous voici au cœur d'un projet colonial tout à la fois autoritaire et libéral.

5 Ainsi, loin de l'image romantique propre aux premiers XIX ${ }^{e}$ siècle, le projet colonial recèle d'emblée cette conception libérale, selon laquelle il est possible d'amender le jeune colon par la terre et d'amender la terre par le travail du colon. De la même manière, il sera possible de faire fructifier les fruits de la colonie tout en améliorant le sort des enfants, sous la promesse d'une ascension sociale au sein de cette société coloniale neuve. L'utilité sociale de la colonisation est le nœud où se joignent sollicitude et intérêt, protection et raison d'État. C'est aussi là que cesse l'utopie pour laisser la place au réalisme colonial, car les sociétés coloniales s'avèrent finalement extrêmement hiérarchisées et violentes. L'envers de l'idéal colonial reste la relégation des indésirables et autres "mauvaises graines » au-delà des mers, selon le principe du bagne ${ }^{3}$. Au point que l'écart entre les visées humanistes des protecteurs de l'enfance et les conditions concrètes de l'exercice du pouvoir colonial paraît inouï de cynisme, dont l'exemple le plus marquant est peutêtre celui du Goulag soviétique étudié par Marta Craveri et Anne-Marie Losonczy.

\section{La place de l'enfance dans le projet colonial : politiques, acteurs, institutions}

Derrière les oripeaux du projet de civilisation, l'enfant apparaît bien comme un instrument placé au cœur du projet colonial. Ce dernier ne suppose pas simplement une exploitation des ressources, mais une emprise politique et culturelle de la "mère patrie " sur un vaste territoire composé de "petites patries » qu'il s'agit d'enjoindre à adhérer à l'idée d'Empire. Au-delà des discours philanthropiques, l'enfance est progressivement considérée comme un vecteur de la colonisation, au nom de son utilité économique et politique. En effet, l'enfant est avant tout une force de travail en devenir, à même de mettre en valeurs les richesses coloniales. Ellen Boucher montre bien qu'il s'agit de confier aux futurs colons blancs la majeure partie du foncier agricole rhodésien, lequel, 
en aucun cas, ne peut échoir à des Africains. L'enfant, en outre, est considéré comme le futur citoyen colonial. Certains prétendent même former, avec les enfants du petit peuple des grandes villes britanniques, les futures élites ultramarines. Les jeunes métis, dont Sarah Heynssens décrit la trajectoire chaotique, sont promis à un meilleur sort que les autochtones rwandais. Le colonisateur belge leur réserve une place de choix dans la société coloniale, dans un élan teint de méfiance à l'égard de la subversion politique que ces « sangs mêlés » pourraient incarner alors que l'indépendance gronde. Yves Denéchère souligne, quant à lui, la valeur postcoloniale des enfants eurasiens dont on imagine qu'ils permettront de perpétuer le lien intime entre l'ancienne métropole et la jeune nation indépendante.

7 Ce pouvoir colonial n'est pas monolithique. Différents acteurs se penchent sur le problème de l'enfance et accompagnent son exil. L'État, bien sûr, est au centre des dispositifs coloniaux. Il fixe le cadre territorial et légal de la migration forcée, qu'il s'agisse de dépayser ou de rapatrier. L'État règle également le statut juridique de ces enfants déplacés : citoyens ou indigènes, ce qui a des conséquences fondamentales sur leurs capacités juridiques et, in fine, sur leurs possibilités d'agir. Mais la puissance publique est rarement maîtresse d'œuvre dans ces migrations et les modalités institutionnelles de prise en charge qui s'en suivent. De nombreuses organisations philanthropiques, agences sociales, associations, congrégations religieuses opèrent sur le terrain, collectant des fonds, organisant les déplacements, édifiant des institutions résidentielles ou des réseaux de familles d'accueil. Leur désintéressement n'est bien souvent qu'un argument rhétorique, participant d'une posture humanitaire qui, au xx siècle, va s'avérer de plus en plus payante politiquement. Malgré des affiliations idéologiques distinctes, les convergences philanthropiques sont capables d'agencer les intérêts des uns et des autres, de former des coalitions de causes. Entrepreneurs de morale y croisent réformateurs sociaux, hommes d'Église y croisent médecins hygiénistes. De fait, les nouveaux experts de l'enfance, démographes, médecins, psychologues et pédagogues prennent part à ces entreprises, comme le signale Ellen Boucher, qui rappelle que les experts des sciences du psychisme ont largement tempéré les ardeurs des sociétés caritatives, en marquant les limites du potentiel éducatif des enfants issus des classes populaires. Enfin, ces déplacements d'enfants acquièrent une portée transnationale au $\mathrm{xx}^{\mathrm{e}}$ siècle, comme l'indique Joëlle Droux. Prendre en charge les mineurs ressortissants étrangers avec les mêmes égards que les nationaux, « renationaliser » les apatrides au lendemain de la dislocation des empires continentaux en Europe constituent une tâche délicate qui témoigne de la difficulté de penser les droits de l'enfant de manière universelle et non nationaliste.

Orphelinats, écoles professionnelles, placement familial, différentes modalités de prise en charge s'offrent au choix des acteurs de cette entreprise migratoire. On pourrait opposer deux modèles : le premier, résidentiel, se donne pour objectif d'acculturer radicalement ; le second, de placement familial ou professionnel, d'assimiler. Ces deux méthodes rencontrent des obstacles : on dénonce l'exploitation des enfants placés chez des colons fermiers, comme le souligne Naomi Parry; on constate des résistances à la discipline institutionnelle, comme le rappelle Yves Denéchère. Dans les deux cas, la frontière entre un traitement sévère, jugé nécessaire à la resocialisation de jeunes marqués par la précarité économique et affective, et les violences institutionnelles, avec le cas spécifique des abus sexuels, est ténue. Et si le corps de l'enfant des colonies est touché par cette violence, ce n'est pas par accident de l'histoire. Au-delà des dispositifs institutionnels et 
des motivations des acteurs de la protection de l'enfance coloniale, le processus même de civilisation est travaillé de l'intérieur par la question du corps, de la race, du sexe et de la filiation, autant de tabous coloniaux que l'analyse historique met au jour.

\section{Les politiques coloniales entre gestion des populations et politisation de l'intime : race, corps, sexe et filiation}

On assiste en effet, à travers les politiques coloniales $d u x^{e}$ siècle, à un double mouvement qui marque l'avènement d'un pouvoir politique qui prend pour objet les populations et saisit les individus dans leur corporéité. Cette «biopolitique » s'applique aux corps selon cette double échelle sociale et individuelle ${ }^{4}$. D'une part, la gestion des populations, au nom d'une démographie coloniale qui fait office de nouvelle expertise, justifie des déplacements forcés, dont certains sont massifs, en particulier au sein de l'Empire britannique, ainsi qu'une attention portée à la question des hybridités « raciales ». D'autre part, on assiste à une politisation de l'intime : corps, sexe, filiation et race deviennent des catégories et des objets d'intervention publique, le creuset dans lequel se forment l'homme-la femme colonial- $\mathrm{e}^{5}$.

$\mathrm{Xx}^{e}$ siècle décrit dans ces pages est le troisième temps de la colonisation, celui de la domination culturelle des colonies, qui fait suite aux phases de conquête militaire et d'établissement de l'exploitation économique. L'emprise du pouvoir passe à la fois par la constitution de populations classifiées et par l'incorporation de l'identité culturelle coloniale ${ }^{6}$. L'enfance est intéressante à cet égard: qu'il s'agisse de jeunes orphelins blancs, de jeunes indigènes "ensauvagés " ou de métis, on la désigne comme une population « désaffiliée » dont on peut alors se saisir pour la déplacer contre son gré et la mettre au service d'un projet de peuplement. Plus encore, l'enfance offre aussi la possibilité de forger une culture coloniale, qu'il s'agisse de former de jeunes colons à la condition «blanche » dans les colonies africaines ${ }^{7}$, ou d'acculturer de jeunes indigènes mis au contact de la culture dominante. Dans cette entreprise, l'éducation scolaire et, souvent, religieuse, occupe une place fondamentale, car elle permet non seulement d'extirper un enfant de sa culture d'origine, mais de le faire adhérer à de nouvelles valeurs, le rendant étranger à sa propre culture. La honte des origines, évoquée par Sarah Heynssens, est un sentiment terriblement éloquent à cet égard.

Bien évidemment, la question raciale est au cœur des politiques coloniales : il s'agit toujours d'établir et de préserver la supériorité de la « race blanche » sur les indigènes. Mais le clivage n'est pas si net. Comme l'explique Ellen Boucher, au moment colonial dont on parle ici, le $\mathrm{xx}^{\mathrm{e}}$ siècle, il n'y a plus de tenants d'une explication strictement biologique de la race qui garantirait une distinction «naturelle» et une hiérarchie raciale incontestable. Selon une vision néo-lamarkienne, la race est une combinaison entre la biologie et le « milieu ». Ce basculement paradigmatique est d'importance : dès lors que la race relève à la fois du sang et de la culture, les moyens pour la "préserver » ou la "défendre ", comme l'expriment les observateurs de l'époque, sont au croisement d'un contrôle de la sexualité et d'une gestion des environnements socio-culturels. Et s'agissant des enfants des colonies, il devient dès lors possible d'inculquer les valeurs et les modes de vie se rapportant à la race "française », "britannique ", ou, plus généralement, " occidentale ». La race devient un fait pédagogique, elle se façonne. 
D'où l'importance du "problème des mulâtres ", du cas des métis qu'abordent Yves Denéchère et Sarah Heynssens, dans la ligne des travaux d'Emmanuelle Saada ${ }^{8}$. Car les métis représentent avant tout une menace pour l'ordre colonial en incarnant une hybridité qui brouille les hiérarchies raciales, trouble le droit civil (quelle filiation?), met en jeu les droits politiques, entre indigénat et citoyenneté9. Ils sont les marqueurs d'une sexualité coloniale subversive. Les métis, d'ailleurs, ont suscité l'inquiétude d'experts de l'enfance comme Georges Heuyer, qui pointait leur «inadaptation " potentielle ${ }^{10}$. On craint, en Rhodésie, une collusion de "race » et de "classe ", le métissage devenant le symbole d'une alliance des dominés contre une conception bourgeoise de l'Empire. Partout, on s'inquiète de la sédition coloniale venue de cette classe de l'entre-deux. Cependant, dans le cas du Ruanda-Urundi, Sarah Heynssens indique que cette classe distincte issue du processus de colonisation incarne aussi, paradoxalement, l'affirmation des bénéfices mutuels du multiculturalisme dont le colonisateur tente de tirer parti, dans l'espoir de former une nouvelle élite coloniale.

13 À l'image du contrôle de la sexualité dont témoigne une police des mœurs très active ${ }^{11}$, les entreprises d'acculturation coloniale sont empreintes d'une volonté de régulation de l'intimité. Les enfants, dans les institutions, font l'objet d'une sollicitude particulière quant à l'hygiène, à la nourriture, aux vêtements, à l'établissement d'une temporalité réglée, comme le soulignent Marie-Pierre Bousquet et Yves Denéchère. La formation d'une identité commune au sein de l'Empire nécessite de soumettre les individus déviants à l'empire des habitudes, lesquelles rompent définitivement tout lien réel avec les origines. Et nul doute que le corps représente à cet égard un site stratégique d'acculturation. Non seulement le corps colonisé est renvoyé aux stéréotypes anthropologiques qu'a construits et véhiculés la culture coloniale, mais il devient l'objet d'une intervention permanente, de manière à le dresser comme on le ferait d'un animal sauvage, à le soumettre à la discipline du colonisateur blanc à des fins de domestication. Le concept de genre, peu évoqué dans ces articles, pourrait offrir à ces études un éclairage supplémentaire, qui introduirait un élément de complexité dans l'analyse de la domination coloniale et de ses politiques migratoires, tant il est vrai que la domination masculine irrigue l'idéologie coloniale ${ }^{12}$.

\section{Expériences coloniales de l'enfance, mémoires coloniales, reconnaissance postcoloniale}

14 Si la question de l'intime est placée sous l'œil du pouvoir colonial, elle n'en reste pas moins un continent obscur, qui renvoie à la subjectivité des enfants eux-mêmes. Qu'en est-il de leur expérience coloniale ? Qu'en est-il de la mémoire coloniale de l'enfance ? Et quels sont les enjeux postcoloniaux de cette histoire des enfants déplacés?

L'expérience des acteurs reste, spécialement lorsqu'il s'agit des enfants, un point de fuite pour l'historien. Qu'ont réellement vécu ces enfants transportés et comment ont-ils vécu ces bouleversements géographiques et culturels? Les auteurs de ce volume insistent avec raison sur la souffrance qui a résulté de ces expériences de déportation et du traumatisme qui a pu en résulter. Marta Craveri et Anne-Marie Losonczy décrivent bien cette «empreinte traumatique " ayant marqué les corps et les esprits des enfants du Goulag soviétique et dont un effet immédiat a été, paradoxalement, l'oubli. S'appliquant à des individus vulnérables ou définis comme tels, l'expérience du déracinement a en effet été 
vécue par les enfants de manière passive et douloureuse, sans qu'il soit souvent possible de résister aux injonctions de leurs "bienfaiteurs", et ce pour différentes raisons: incapacité juridique, précarité économique, soumission symbolique attendue de la jeunesse, construction de hiérarchies ethnico-raciales...

Mais à travers les récits que relatent les auteurs, les jeunes ont fait preuve de différentes formes de résistances. Les «hasards de l'histoire» ont pu permettre aux jeunes d'échapper à un sort plus funeste encore, et parfois de bénéficier réellement d'une mobilité sociale salvatrice. Les trajectoires biographiques d'un $\mathrm{xx}^{\mathrm{e}}$ siècle chaotique sont aussi faites de ces petits miracles dont ont su tirer parti les jeunes. De plus, le regard des enfants sur le drame et la violence a pu permettre des déplacements de sens. Transformer la tragédie en "aventure", débusquer le grotesque dans l'autorité outrancière des institutions, se réinventer une famille parmi ses compagnons d'infortune, faire de l'ailleurs sa maison, telles ont pu être, au creux de l'imaginaire enfantin, les manières de dédramatiser l'insupportable.

Depuis deux décennies, il s'est agit de témoigner de la souffrance, des outrages souvent infligés par les institutions coloniales à des individus d'autant plus vulnérables qu'il s'agissait d'enfants. Aussi, les méandres de la mémoire infantile des colonies sont-ils extrêmement sinueux. Il faut bien considérer, d'abord, que les «cadres sociaux de la mémoire ${ }^{13}$ » sont particulièrement prégnants s'agissant de l'expérience coloniale. Il existe bien une mémoire collective de la colonisation qui peut faire écran à la mémoire individuelle, et en particulier enfantine, de cette expérience. Plutôt que de relever d'une expérience intime, la mémoire de l'enfance coloniale est chargée d'un sens politique fort, marqué du sceau de la violence qui conduit à l'oubli, puis à des phénomènes de "remémoration tardive », comme l'expliquent Marta Craveri et Anne-Marie Losonczy. Le "devoir de mémoire " peut aussi, comme l'indique Yves Denéchère, être suscité par un effet générationnel : les descendants réclamant un « récit des origines ». Mémoire obérée par l'indicible épreuve du déracinement, mémoire réinventée face aux aléas de la grande histoire comme du roman familial, impossible mémoire d'une colonisation qui cherchait justement à effacer les origines, la mémoire coloniale est double, voire schizophrène. Elle relève à la fois du récit, agencement de la réalité qui lui donne sens, mais doit répondre également à un impératif de "vérité", lequel ouvre seul à la possibilité d'une reconnaissance postcoloniale.

Se sont ainsi construites des postures politiques divergentes à l'égard de ces expériences juvéniles de la déportation coloniale. D'une part, la reconnaissance du statut de victime s'est inscrite dans ce grand mouvement victimaire qui a marqué les vingt dernières années. Les collectifs se sont formés, notamment grâce aux réseaux de communication numériques, recueillant les témoignages, revendiquant une expertise profane parfois concurrente de celle des historiens professionnels, réclamant le plus souvent des excuses officielles de la part des gouvernements, voire des dédommagements financiers ou la restitution de droits et de biens ancestraux, comme le montre bien Naomi Parry au sujet des Aborigènes australiens ${ }^{14}$. Cette position de victime, devenue, après des années d'indifférence, relativement lucrative politiquement, a pesé sur les débats, et produit de nouvelles hiérarchies de légitimité en décrivant qui pouvait (ou pas) se revendiquer de ce statut. D'autres formes de revendications existent également, mettant de l'avant la réappropriation de leur histoire par les enfants déplacés des colonies. Il s'agit de faire de l'expérience coloniale un élément de la construction d'une identité métissée, créole, hybride. Cette dernière ne donne pas nécessairement lieu à un pardon ou à un 
dédommagement qui solderait les comptes de la mémoire, elle suscite une remise en cause de l'hégémonie culturelle du colonisateur et revendique un statut politique dans le présent, en suivant la voie ouverte par les études postcoloniales dont une figure de proue reste E. Saïd ${ }^{15}$.

Au nom de l'enfance, érigée en cause " universelle », le pouvoir colonial s'est appliqué à façonner les sociétés indigènes, organisant la migration de dizaines de milliers d'enfants contre leur gré, sans égards pour les racines culturelles des jeunes ainsi (dé)placés. Il s'agit ici de reconsidérer cette histoire croisée, où le métissage et la «créolisation » ne sont pas conçus comme des plaies à panser mais comme des ressources pour construire un monde postcolonial ${ }^{16}$.

\section{NOTES}

1. PERrot Michelle, "La ségrégation de l'enfance au $\mathrm{XIX}^{\mathrm{e}}$ siècle », Psychiatrie de l'enfant, XXV, 1, 1982, p. 179-207.

2. VARGA Donna, « Look-normal : The colonized child of developmental science », History of Psychology, 14, 2, 2011, p. 137-157.

3. Kalifa Dominique, Biribi: les bagnes coloniaux de l'armée française, Paris, Perrin, 2009 ; T HÉNAULT Sylvie, Violence ordinaire dans l'Algérie coloniale: Camps, internements, assignations à résidence, Paris, Odile Jacob, 2012.

4. Foucault Michel, Naissance de la biopolitique. Cours au Collège de France (1978-1979), Paris, Gallimard/Le Seuil, 2004.

5. CAMISCIOLI Elisa, Reproducing the French race : immigration, intimacy, and embodiment in the early twentieth century, Durham (N. C.), Duke University Press, 2009.

6. Mudimbe V. Y., The Invention of Africa: Gnosis, Philosophy and the Order of Knowledge, Bloomington, Indiana University Press, 1988.

7. Soulignons l'intérêt que représente le nouveau chantier historiographique des Whiteness Studies, études critiques sur l'identité « blanche ».

8. SAADA Emmanuelle, Les enfants de la colonie. Les métis de l'Empire français entre sujétion et citoyenneté, Paris, La Découverte, 2007.

9. Au point qu'en Algérie, comme l'explique Christelle Taraud, les métis ont été complètement occultés dans les discours publics. TARAuD Christelle, «Les yaouleds : entre marginalisation sociale et sédition politique », Revue d'histoire de l'enfance "irrégulière », $\mathrm{n}$ - 10, 2008, p. 59-74.

10. HeUYeR Georges et LAUTMANN Françoise, "Troubles du caractère et inadaptation sociale chez les enfants métis", Archives de médecine des enfants, tome 40, $\mathrm{n}^{\circ} 9$, septembre 1937, p. 553-564.

11. TRACOL-HUYNH Isabelle, «Encadrer la sexualité au Viêt-Nam colonial : police des mœurs et réglementation de la prostitution (des années 1870 à la fin des années 1930) », Genèses, 86, 1, 2012, p. 55-77. 
12. BARThélémy Pascale, CAPdevila Luc, ZANCARIni-fournel Michelle, "Colonisations ", Clio. Histoire, femmes et sociétés, 33, 2011; Hugon Anne, Histoire des femmes en situation coloniale : Afrique et Asie, $\mathrm{XX}^{e}$ siècle, Paris, Karthala, 2004 ; TARAUD Christelle, La prostitution coloniale : Algérie, Tunisie, Maroc, 1830-1962, Paris, Payot, 2003.

13. HalbWACHS Maurice, Les Cadres sociaux de la mémoire, Paris, Albin Michel, 1994 (1925).

14. Engel Madeline H., Phillips Norma Kolko et DellaCAVA Frances A., «Indigenous Children's Rights: A Sociological Perspective on Boarding Schools and Transracial Adoption ", International Journal of Children's Rights, 20, 2, 2012, p. 279-299.

15. SAID Edward-W., L'orientalisme : L'Orient créé par l'Occident, Paris, Le Seuil, 2005.

16. ApPADURA Arjun, Après le colonialisme. Les conséquences culturelles de la globalisation, Payot, 2005. MBEMBE Achille, Sortir de la grande nuit. Essai sur l'Afrique décolonisée, Paris, La Découverte, 2010.

\section{AUTEUR}

\section{DAVID NIGET}

CERHIO, université d'Angers. 